\title{
Preliminary evidence for signature vocalizations among free-ranging narwhals (Monodon monoceros) ${ }^{\text {a) }}$
}

\author{
Ari D. Shapiro ${ }^{\text {b) }}$ \\ Biology Department, Woods Hole Oceanographic Institution, MS \#50, Woods Hole, Massachusetts 02543
}

(Received 14 March 2006; revised 9 June 2006; accepted 21 June 2006)

\begin{abstract}
Animal signature vocalizations that are distinctive at the individual or group level can facilitate recognition between conspecifics and re-establish contact with an animal that has become separated from its associates. In this study, the vocal behavior of two free-ranging adult male narwhals (Monodon monoceros) in Admiralty Inlet, Baffin Island was recorded using digital archival tags. These recording instruments were deployed when the animals were caught and held onshore to attach satellite tags, a protocol that separated them from their groups. The signature content of two vocal categories was considered: (1) combined tonal/pulsed signals, which contained synchronous pulsatile and tonal content; (2) whistles, or frequency modulated tonal signals with harmonic energy. Nonparametric comparisons of the temporal and spectral features of each vocal class revealed significant differences between the two individuals. A separate, cross-correlation measure conducted on the whistles that accounted for overall contour shape and absolute frequency content confirmed greater interindividual compared to intraindividual differences. These data are consistent with the hypothesis that narwhals produce signature vocalizations that may facilitate their reunion with group members once they become separated, but additional data are required to demonstrate this claim more rigorously. (C) 2006 Acoustical Society of America. [DOI: 10.1121/1.2226586]
\end{abstract}

PACS number(s): 43.80.Ka, 43.80.Ev [WAA]

Pages: 1695-1705

\section{INTRODUCTION}

Signature vocalizations of animals acoustically encode individual or group identity and are characterized by unique sets of spectral and/or temporal attributes. The specific acoustic features required to distinguish between individuals according to their vocalizations have been measured in a host of taxa [e.g., birds: macaroni penguins (Eudyptes chrysolophus), Searby et al., 2004, chiropterans: evening bats (Nycticeius humeralis), Scherrer and Wilkinson, 1993, canids: timber wolves (Canis lupus), Goldman et al., 1995, primates: common marmosets (Callithrix jacchus), Jones et al., 1993, pinnipeds: subantarctic fur seals (Arctocephalus tropicalis), Charrier et al., 2001, 2003, cetaceans: bottlenose dolphins (Tursiops truncatus), Janik, 1999, Watwood et al., 2005]. Playback experiments have demonstrated that animals can recognize signature signals and have illustrated the diversity of contexts in which signature vocalizations are used, including facilitating recognition between an infant and one or both of its parents [cliff swallows (Hirundo pyrrhonota): Stoddard and Beecher, 1983, tree swallows (Tachycineta bicolor): Leonard et al., 1997, Mexican free-tailed bats (Tadarida brasiliensis mexicana): Balcombe, 1990, fur seals (Arctocephalus tropicalis): Charrier et al., 2001, 2003, bottlenose dolphins: Sayigh et al., 1998, Janik et al., 2006], mate-pair recognition [king penguins (Aptenodytes patagonicus): Lengagne et al., 2000], and group affiliation associated with territorial de-

\footnotetext{
${ }^{\mathrm{a})}$ Portions of this work were presented in "Vocal behavior of free-ranging Arctic narwhals (Monodon monoceros)," Proceedings of the 16th Biennial Conference on the Biology of Marine Mammals, San Diego, CA, December 2005.

b) Author to whom correspondence should be addressed. Electronic mail: ashapiro@whoi.edu
}

fense (North American bullfrog (Rana catesbeiana): Bee and Gerhardt, 2002, Arctic foxes (Alopex lagopus): Frommolt et al., 2003). There are selective benefits for the signals produced in these contexts. Recognition is very important when one or both parents must allocate a finite amount of resources to their offspring, a scenario in which confusion is associated with high fitness costs. Mates or groups of individuals that consistently defend one another, their young, or their territory can benefit from individual or group recognition because it provides a system for remembering with whom they have shared mutual investments.

The proximate methods for achieving signature recognition can include imprinting, habituation, associative learning, and vocal learning. Vocal learning occurs when the respiratory, phonatory, and/or filter systems are employed to render signals more or less similar to acoustic models that are encountered through experience with other individuals (Janik and Slater, 1997, 2000). Contact calls, generally used by animals when they become separated from their social partner(s) or group to first locate one another and then mediate reunion, contain signature content in certain species and appear to be vocally learned. When placed in social groupings of unfamiliar individuals, the contact calls of male budgerigars (Melopsittacus undulatus), for example, initially converged and subsequently underwent continuous and synchronous changes (Farabaugh et al., 1994). Evidence is accumulating for some species of nonhuman primates to possess vocal plasticity during adulthood despite its apparent absence during development (see review by Egnor and Hauser, 2004). Male chimpanzees (Pan troglodytes) produce pant hoots, long-distance vocalizations that seem to function in maintaining contact with and attracting allied individuals 
(Mitani and Nishida, 1993). Pant hoot convergence was observed among both chorusing dyadic pairs (Mitani and GrosLouis, 1998) and larger groups containing 3-11 adult males (Marshall et al., 1999). Similarly, several spectral and temporal parameters of the contact calls among pygmy marmosets (Cebuella pygmaea), referred to as trills, underwent parallel or convergent shifts between new adult mate pairs (Snowdon et al., 1997; Snowdon and Elowson, 1999). Comparable observations were made when two naïve pygmy marmoset social groups of mixed-age composition were introduced (Elowson and Snowdon, 1994).

In principle, it is possible to conclude that signals contain signature content by demonstrating more interindividual than intraindividual variability. To offer sufficient proof, this result must be shown for at least several exemplars of the signature signals of each of several individuals. One of the most striking examples of signature vocalizations is found among bottlenose dolphins whose signature whistles, first identified by Caldwell and Caldwell (1965), appear to function as vocally-learned contact calls. Among bottlenose dolphins, whistle imitation appears to be an important agent in the ontogeny of this signature vocalization (Sayigh et al., 1990, Miksis et al., 2002) and in social communication both in captivity (Tyack, 1986) and in the wild (Janik, 2000, Fripp et al., 2005). Bottlenose dolphin mother-calf pairs were more likely to orient towards one another's signature whistles when separated from each other and temporarily restrained than those of other individuals of the same corresponding age cohort (Sayigh et al., 1998, Janik et al., 2006). Captive animals produced their signature whistles most often when they separated themselves voluntarily and spontaneously from their mixed-age group by swimming into an adjacent tank compared to when they were swimming together (Janik and Slater, 1998). The remaining dolphins were also more likely to produce their signature whistles when an individual left the main tank. Finally, adult males that had strong social bonds with another male were most likely to use signature whistles when they were separated either due to temporary, artificial restraint or voluntarily when they were free-ranging, presumably to facilitate an eventual reunion (Watwood et al., 2005). Collectively, these studies reveal the importance of signature whistles in maintaining contact between bottlenose dolphin individuals in artificial and natural settings alike and in both involuntary and voluntary contexts.

Despite some debate (see McCowan and Reiss, 1995, 2001; Janik, 1999 for discussion; Smolker and Pepper, 1999), the studies just described have helped to solidify the case for signature whistles in bottlenose dolphins and suggest the possibility of signature vocalizations among other long-lived, social odontocetes in an underwater environment where acoustic signaling is the most reliable and efficient form of communication. In addition, signature information can also be encoded at the group level, which can form the basis for acoustic badges of membership (e.g., Boughman, 1997). For example, five of six shared call types among killer whales (Orcinus orca) revealed distinctive structural differences between matrilineal units (Miller and Bain, 2000) and it is possible that other signature details allow differentiation between individual animals as well (Nousek et al., in press). Cultural divergence of discrete call types appears to account for some of the subtle differences in the temporal and spectral features across these matrilineal units and even within pods (Deecke et al., 2000).

The principal challenge for studying the signature signals of marine mammals involves the difficulty of assigning vocalizations in the wild unambiguously to the individual animal that produced them. In this manuscript, the possibility of signature signals among free-ranging narwhals (Monodon monoceros) was examined by recording the acoustic activity of two individuals with digital archival tags. These gregarious, long-lived Arctic odontocetes migrate distances of thousands of kilometers in large numbers with subpopulations moving in a coordinated fashion (Hay and Mansfield, 1989; Dietz and Heide-Jørgensen, 1995; Laidre et al., 2004). They travel in groups that are often sex segregated and range in size from a few animals to dozens of individuals, although the stability or fluidity and interconnectedness of these assemblages remain unknown (reviewed in Hay and Mansfield, 1989).

Narwhals produce echolocation clicks with repetition rates between 2 and >500/s (Ford and Fisher, 1978, Møhl et al., 1990), maximum frequencies reaching at least $160 \mathrm{kHz}$ (Miller et al., 1995) and maximal source levels reaching $218 \mathrm{~dB}$ re $1 \mu \mathrm{Pa}$ (Møhl et al., 1990). Miller et al.(1995) arbitrarily divided clicking into the two categories of train clicks produced at $\leqslant 30$ clicks/s and burst clicks produced at $\geqslant 40$ clicks/s. Pulsatile sounds featuring a repetition rate high enough to possess a tonal character with harmonically related sidebands (see Watkins, 1967) were called longer click series by Watkins et al. (1971) and pulsed tones by Ford and Fisher (1978). Characterized as narrow-band, these signals had durations between 0.56 and several seconds and spectral energy ranging from $500 \mathrm{~Hz}$ to $24 \mathrm{kHz}$. The repetition rate was generally constant although Watkins et al. (1971) reported a tendency for the repetition rate of these vocalizations to increase at the very beginning and slow down towards the end. In this manuscript, these signals will be referred to as combined tonal/pulsed signals. Finally, narrow-band, frequency modulated (FM) whistles have been described that generally last $<1.0 \mathrm{~s}$ (range: $0.1-6.0 \mathrm{~s}$ ) and have a frequency range between $300 \mathrm{~Hz}$ and $18 \mathrm{kHz}$ (Ford and Fisher, 1978; Møhl et al., 1990).

Although Ford and Fisher (1978) did not find any evidence for signature content among whistles, they speculated that the different pulsed tones in their recordings were produced by separate individuals as signature calls in a social context. They recorded series of the same tone growing louder and then softer, concluding that this resulted from one individual producing each series as it approached and then swam past a stationary hydrophone. This possibility was not conclusive since groups of animals were swimming by the recorder and multiple individuals could have been producing each tone. In addition, no data on differences in acoustic parameters were available to quantify the distinctiveness of the calls. In this study, we examined the possibility of signature vocalizations among free-ranging narwhals more closely. The results support this hypothesis for both combined tonal/pulsed signals and whistles, suggesting a social 


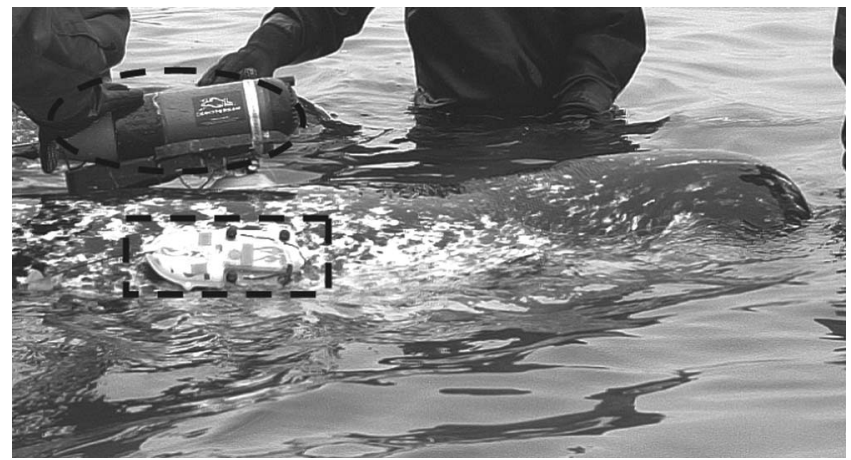

FIG. 1. Narwhal shown with Crittercam (contained within the dashed ellipse) and DTAG (contained within the dashed rectangle) attached immediately before release. Photograph courtesy of Rune Dietz.

function for vocal production that is distinctive either at the individual or group level. Further work is recommended to confirm signature vocal production among additional animals and to ascertain the natural function of these vocalizations in the wild.

\section{METHODS}

\section{A. Study area}

Field work was conducted from 8-23 August 2004 at Kakiak Point, Admiralty Inlet on Baffin Island in Nunavut, Canada $\left(73^{\circ} 40^{\prime} \mathrm{N}, 86^{\circ} 40^{\prime} \mathrm{W}\right)$. The inlet has a maximum depth of $720 \mathrm{~m}$. Groups of narwhals ranging from approximately 5-30 individuals (pers. obs.) traveled into the inlet at this time of year once the ice had mostly melted. The field camp occupied a position about $500 \mathrm{~m}$ from a site used intermittently by the Inuit to hunt narwhals.

\section{B. Equipment}

This experiment employed a digital archival tag (DTAG) developed by Johnson and Tyack (2003) featuring a single hydrophone, pressure and temperature sensors, and a triaxial accelerometer and magnetometer, which recorded to flash memory. The sampling rate of the hydrophone was set to $96 \mathrm{kHz}$ while the other sensors sampled at $50 \mathrm{~Hz}$. A 16 bit ADC was used. Sigma delta conversion provided an effective antialiasing filter, dispensing with aliasing caused by energy exceeding the Nyquist frequency of $48 \mathrm{kHz}$. The tag attached noninvasively to individual animals via suction cups and its release was coupled to the release mechanism of the National Geographic Crittercam (see Marshall, 1998) that was deployed simultaneously. A VHF transmitter signaled the location of an attached tag intermittently as the animal surfaced and then regularly once the tag was released and floated to the water's surface.

\section{Capturing and tagging protocol}

The DTAG was deployed in collaboration with a satellite tagging project that required working with the animals onshore. As described by Dietz et al. (2001), a $50 \mathrm{~m}$ long and $10 \mathrm{~m}$ deep black net with $20 \times 20 \mathrm{~cm}$ mesh was oriented perpendicular to the shore and kept afloat with 7-8 white buoys. The net was secured to the shore and in the water.

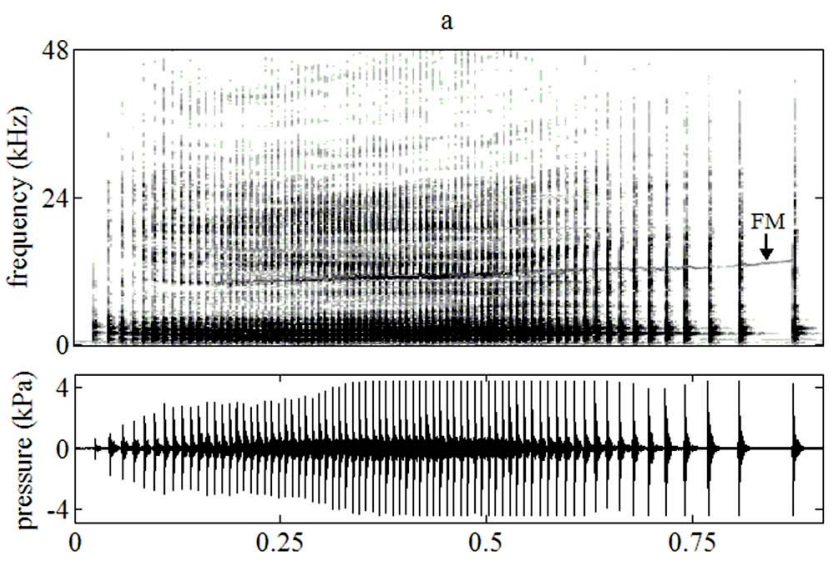

b
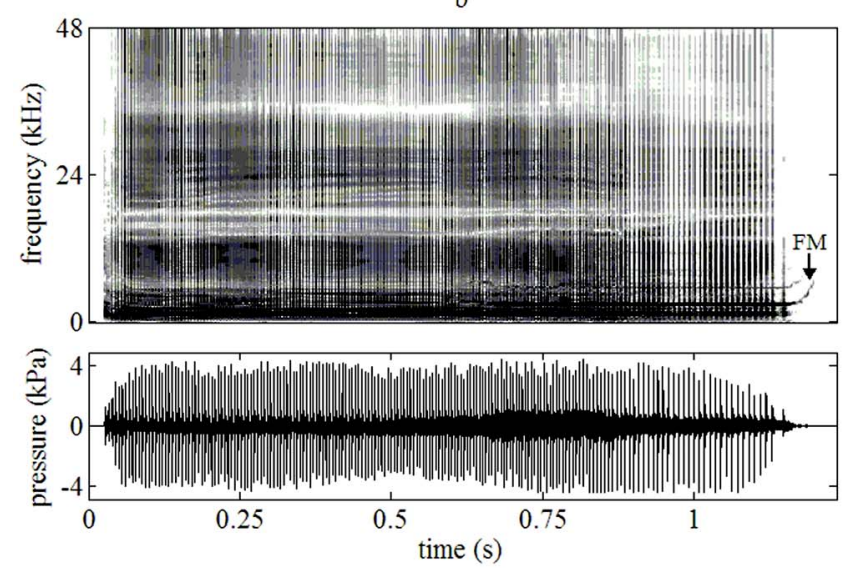

FIG. 2. Sample spectrograms (larger, top plots) and waveforms (smaller, bottom plots) of a combined tonal/pulsed signal produced by individual (a) mm224 and (b) mm226 with a FFT size and frame length of 512 points, $50 \%$ window overlap, and a maximum frequency displayed of $48 \mathrm{kHz}$. The low frequency energy associated with most of the pulses is likely due to the resonance of the air sacs involved in sound production or transmission. The solid arrows in both spectrograms indicate the synchronous FM tonal component produced by the tagged animal.

When weather conditions permitted, the net was deployed and monitored constantly for caught animals, signaled by the submergence of at least one of the buoys. As soon as a whale became entangled, two boats were dispatched immediately to bring the animal to the surface to breathe and tow it to shore with the assistance of a land-based crew hauling on the net. Once an animal was caught, the remainder of its group moved out of visual range, presumably continuing their migration deeper into the inlet. A fluke belt was used to keep the animal ashore and oriented with its head submerged and pointed into the water while its blowhole had access to the air at all times. Three males and five females were captured in all. During satellite tag attachment, blood samples were collected to assess overall health and stress levels.

Just before two of the adult males and one of the adult females were guided back to deeper water, a DTAG was attached to their dorsal sides $\sim 0.5 \mathrm{~m}$ caudal to the blowhole (Fig. 1). These animals were not followed visually once they were released from shore so it was not possible to determine whether they eventually reunited with their group members. The VHF signal was monitored from the field camp on shore using two handheld yagi antennae. Once a regular VHF sig- 
a

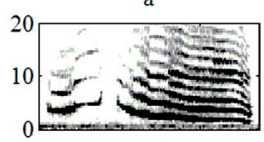

$\mathrm{b}$

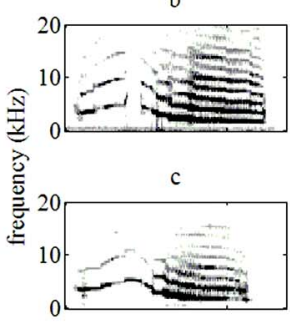

d
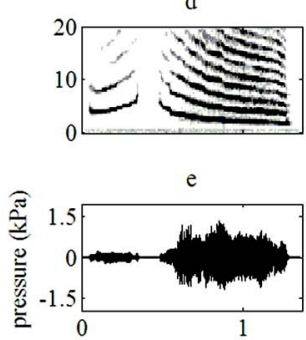

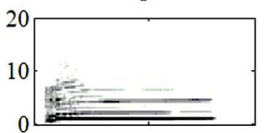

g

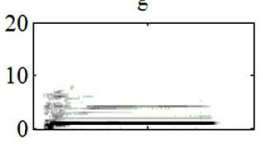

$\mathrm{h}$
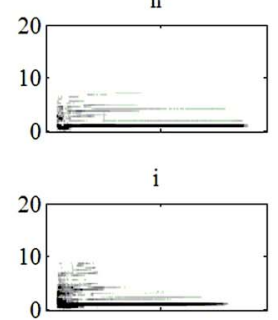

j

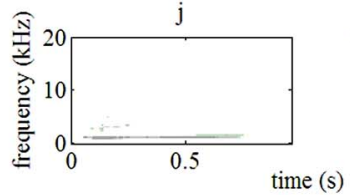

$\mathrm{k}$
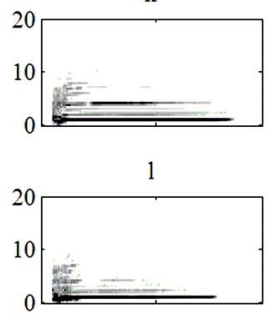

$\mathrm{m}$
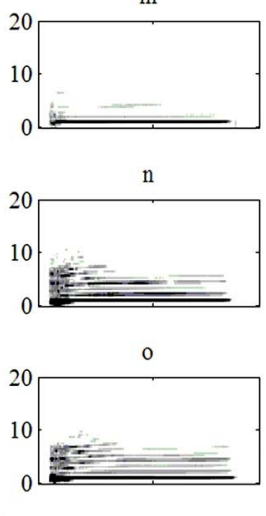

$\mathrm{p}$

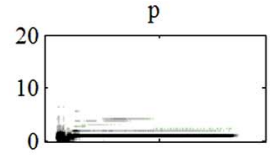

q

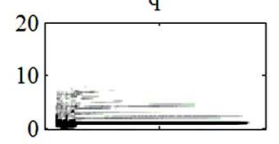

$\mathrm{r}$

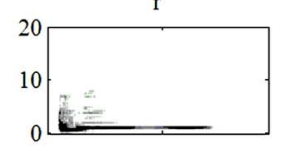

s
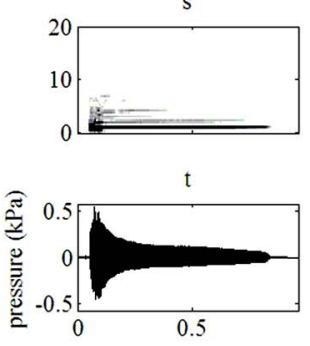

FIG. 3. Spectrogram composite of all four whistles of mm224 (a-d) and 14 of the 17 whistles of $\mathrm{mm} 226$ (f-s) with a FFT size and frame length of 512 points, $50 \%$ window overlap, and a maximum frequency displayed of $20 \mathrm{kHz}$. The remaining 3 whistles of mm226 resembled those displayed here but were excluded for graphical convenience. The waveforms displayed in subplots e and $t$ are of the same whistles used to generate subplots $\mathrm{d}$ and $\mathrm{s}$, respectively. nal was detected from a tag that had released from the animal and the weather permitted, a boat was dispatched for recovery. If the VHF signal grew too faint to detect from shore, tracking was conducted from a higher altitude on the nearby cliffs for improved range. The first tag recorded for $2.54 \mathrm{~h}$ (male $\mathrm{mm} 224$ ), the second tag for $12.14 \mathrm{~h}$ (male mm226), and the third tag was not recovered. These two tagged males entered the inlet two days apart, strongly suggesting that they belonged to different social groups. The data were offloaded and burned to $\mathrm{CD}$ in duplicate in the field.

\section{Vocalization extraction}

The $14.68 \mathrm{~h}$ of recordings were audited by listening to and visually examining the spectrograms in $15 \mathrm{~s}$ segments. Focal (tagged animal) vocalizations were marked according to their starting time and vocal category. It was assumed that vocalizations with a relatively high signal to noise ratio (SNR) belonged to the focal animal and not a neighboring nonfocal animal. Although this assumption could not be verified visually because the tagged narwhals were not followed, it was true for at least the first few dives since no group members were observed in the immediate vicinity. Much softer sounds were often heard on the recordings, presumably from more distant, vocalizing nonfocal animals. The SNR of these focal vocalizations was computed by comparing the root of the mean of the squared pressure (RMS) along the window containing $90 \%$ of the signal energy to a segment of noise of the same duration immediately preceding the signal (Madsen, 2005). The analysis presented here only excluded echolocation clicks, or broadband pulses of energy with interclick intervals usually greater than 100 ms. Nearly all remaining vocalizations were considered that could be divided into the two discrete categories of (1) combined tonal/pulsed signals (Fig. 2), defined as uninterrupted pulsatile vocalizations with a synchronously produced FM tonal component, a low mean interpulse interval (IPI $<13 \mathrm{~ms}$ ), and high pulse number (>49), and (2) whistles (Fig. 3), which were characterized by FM, tonal energy with several harmonics. See the discussion in this manuscript for a comparison of these designations to earlier classification schemata. All vocalizations were saved as separate wav files. Individual mm 224 produced 42 combined tonal/pulsed signals and 4 whistles and mm226 produced 31 combined tonal/ pulsed signals and 17 whistles.

\section{E. Analysis of combined tonal/pulsed signals}

Pulses were located automatically using customized Matlab 7.1 (The MathWorks, Inc.) software that, given a user-provided threshold value, triggered on and marked abrupt peaks in the pressure waveform. Subsequent inspections of all waveforms were made to select undetected and remove erroneously marked pulses. A nonparametric Wilcoxon rank sum test with a Bonferroni correction was used to examine whether the four parameters of average IPI, duration, number of pulses, and pulse repetition rate were significantly distinguishable between the two individuals. The pulse repetition rate and the normalized pulse number were also plotted as functions of the normalized duration to provide a visual means of comparing these sounds.

\section{F. Whistle extraction and analysis}

The fundamental frequency contour of each whistle spectrogram (FFT size and frame length of 2048 points with $50 \%$ window overlap) was traced by hand with customized Matlab software (Fig. 4). One hundred equally spaced points were extracted from these contours and normalized to a time axis between 0 and 1 (see Watwood et al., 2004, 2005). Two tests of similarity were conducted on these whistle contours: 
a

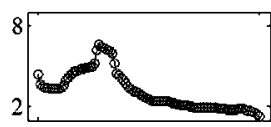

b
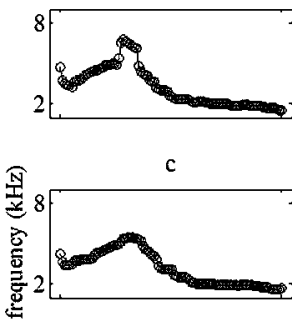

d

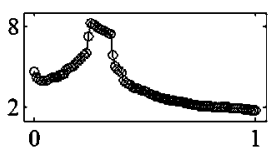

f

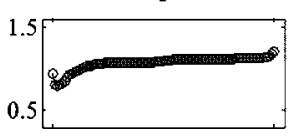

g

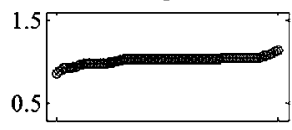

h

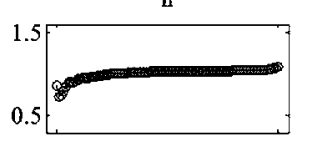

i

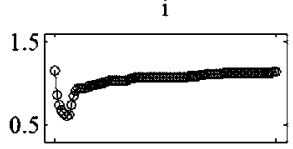

j

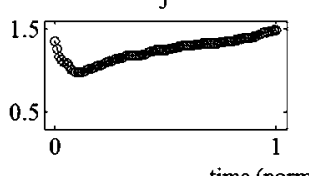

time (normalized units) k

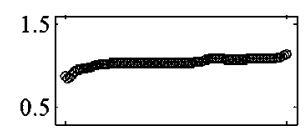

1

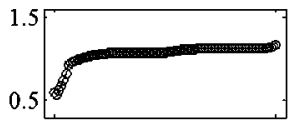

m

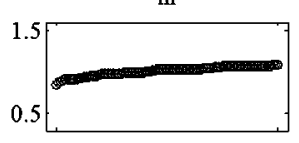

n

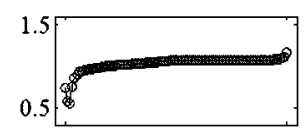

o

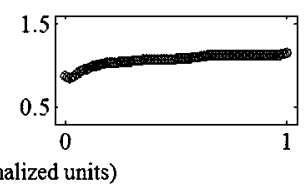

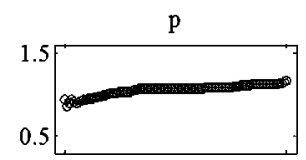

q

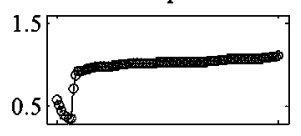

r

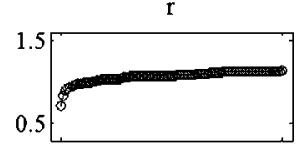

$\mathrm{s}$

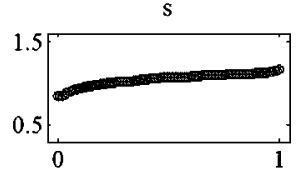

FIG. 4. Digitized traces of the fundamental frequency contours of the whistles displayed in Fig. 3. Each trace is shown with 100 equally spaced points that have been normalized on a horizontal time axis from 0 to 1. Again, panels a-d correspond to the whistles produced by $\mathrm{mm} 224$ and $\mathrm{f}-\mathrm{s}$ to those by $\mathrm{mm} 226$. Note the difference in the frequency ranges for the two individuals.

\section{Nonparametric comparison}

One temporal (original duration before normalization) and five spectral (minimum, maximum, mean, initial, and ending frequencies) features were determined for every whistle (Fig. 5). These parameters were selected because they summarized the timing and coarse frequency content of the whistles. The differences between the finer aspects of the

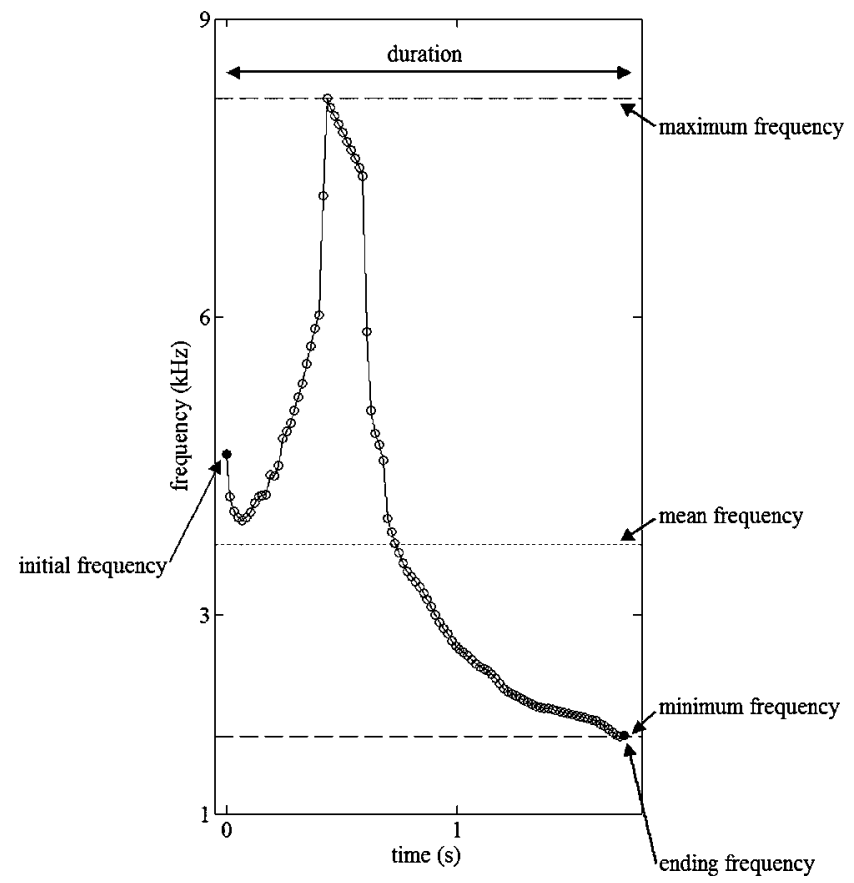

FIG. 5. Illustration of temporal and spectral features extracted from a traced whistle produced by mm224 (subplot d in Figs. 3 and 4). The initial and ending frequencies are indicated by filled circles $(0)$ while the other spectral components are marked with horizontal dashed lines. Note that the mean frequency is closer to the minimum frequency because $\sim 60 \%$ of the whistle's frequency content lies below $3700 \mathrm{~Hz}$. frequency contours were reserved for the cross-correlation comparison. Again, a nonparametric Wilcoxon rank sum test with a Bonferroni correction was used to compare these features between individuals.

\section{Cross-correlation comparison}

Cross-correlation is often used in signal processing as a tool for determining the similarity between two signals. Because the frequency ranges of the whistles from the two individuals were distinct (e.g., the average maximum frequency of mm226 was less than the average minimum frequency of mm224), the cross-correlation measurement used here was designed to account for overall contour shape and absolute frequency content. In the equation

$$
\sum_{i=1}^{100} \frac{\left|\left(f_{A}(i)-f_{B}(i)\right)\left(f_{A}(i)-f_{B_{m}}(i)\right)\right|}{\overline{f_{A}(i)+f_{B}(i)}},
$$

$i$ is the sample number that ranges between 1 and 100, $f_{A}(i)$ and $f_{B}(i)$ correspond to the $i$ th frequency value of contours $A$ and $B$, respectively, and $f_{B_{m}}(i)$ is the $i$ th frequency value of contour $B$ after it has been slid along the frequency axis to minimize the frequency differences between contours $A$ and $B$. These terms are illustrated in Fig. 6. Larger values of this cross-correlation measure indicated greater differences between contours than smaller values. A value of 0 would reveal no difference at all in contour shape. A constrained, nonlinear minimization routine was used to determine $f_{B_{m}}(i)$. The first term in the product of the numerator of (1) is the difference between points along the actual contours normalized in time [Fig. 6(a)]. The second term, however, returns a smaller number if the frequency modulation pattern is similar between the whistles regardless of the absolute frequency offset of the two [Fig. 6(b)]. Whistles therefore could have achieved a higher similarity 


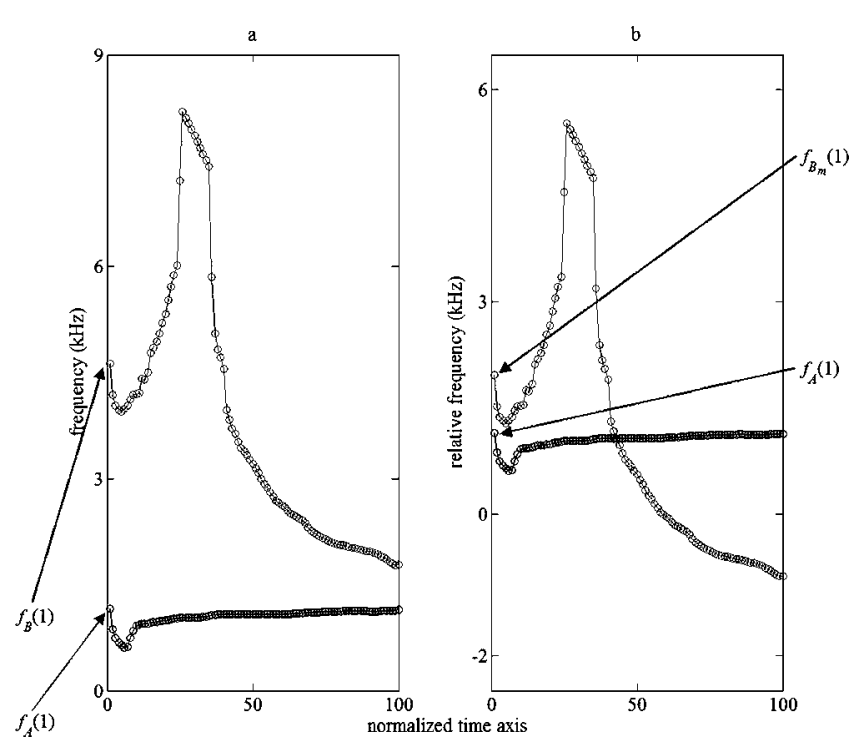

FIG. 6. Illustration of points used for cross-correlation comparison of whistles (see text for the equation). In (a), contour $A$ (darker, from mm226: subplot i in Figs. 3 and 4 ) and $B$ (lighter, from mm224: subplot d in Figs. 3 and 4) are depicted normalized in time with their original frequency content. In (b), contour $B$ has been shifted along the frequency axis to minimize the frequency difference between the two contours. All 100 points along the contours were used to compute Eq. (1).

ranking [a smaller value of (1)] by overlapping in absolute frequency, possessing similar overall contour shapes or both.

\section{RESULTS}

Tables I and II list the summary measurements of the combined tonal/pulsed signals and whistles. With the exception of a single whistle assigned to mm226 with a SNR of $13.9 \mathrm{~dB}$, the remaining vocalizations produced by both animals were characterized by a SNR of at least $28.2 \mathrm{~dB}$. Combined tonal/pulsed signals were produced throughout the water column but tended to concentrate at particular depths (roughly $70 \mathrm{~m}$ for $\mathrm{mm} 224$ and $20 \mathrm{~m}$ for $\mathrm{mm} 226$, Fig. 7). Whistle production occurred between 20 and $100 \mathrm{~m}$ for $\mathrm{mm} 224$ but was confined to the upper $30 \mathrm{~m}$ for mm226 (Fig. 7). Both vocal categories were recorded throughout the diving sequence, indicating that the behavioral or environmental contexts in which these vocalizations occurred were not gen-

TABLE I. Summary statistics of the acoustic features of combined tonal/ pulsed signals.

\begin{tabular}{crrrr}
\hline \hline & Mean & Std. & Min. & Max. \\
mm224, & $n=42$ & & & \\
Duration (s) & 1.6 & 0.7 & 0.6 & 2.7 \\
Average IPI (ms) & 12.9 & 4.3 & 9.0 & 36.1 \\
Number of pulses & 128.1 & 45.4 & 49.0 & 202.0 \\
Pulse repetition rate (pulses/s) & 82.3 & 14.2 & 28.1 & 112.8 \\
& & & & \\
Duration (s) & 1.2 & 0.1 & 1.0 & 1.2 \\
Average IPI (ms) & 6.3 & 0.3 & 5.6 & 6.8 \\
Number of pulses & 186.2 & 9.6 & 156.0 & 201.0 \\
Pulse repetition rate (pulses/s) & 160.8 & 7.4 & 147.5 & 180.5 \\
\hline \hline
\end{tabular}

TABLE II. Summary statistics of the acoustic features of whistles.

\begin{tabular}{rcrrr}
\hline \hline & Mean & Std. & Min. & Max. \\
\hline & mm224, $n=4$ & & & \\
Duration (s) & 1.19 & 0.08 & 1.09 & 1.26 \\
Minimum frequency (Hz) & 1549 & 201 & 1292 & 1775 \\
Maximum frequency (Hz) & 7181 & 1386 & 5460 & 8844 \\
Mean frequency (Hz) & 3638 & 370 & 3405 & 4190 \\
Initial frequency (Hz) & 4773 & 277 & 4496 & 5145 \\
Ending frequency (Hz) & 1572 & 216 & 1292 & 1788 \\
& & & & \\
Duration (s) & 0.78 & 0.04 & 0.68 & 0.85 \\
Minimum frequency (Hz) & 718 & 156 & 360 & 980 \\
Maximum frequency (Hz) & 1177 & 111 & 1095 & 1501 \\
Mean frequency (Hz) & 1012 & 71 & 895 & 1240 \\
Initial frequency (Hz) & 939 & 236 & 641 & 1501 \\
Ending frequency (Hz) & 1160 & 90 & 1095 & 1486 \\
\hline \hline
\end{tabular}

erally restricted to a very narrow depth or time. The two animals responded differently immediately after handling. Many combined pulsed/tonal signals (17 of 42) and one whistle were produced by individual mm224 just after release on his first dive lasting only $10.8 \mathrm{~min}$. Individual mm226 was vocally active, however, between hours 4 and 10 of the deployment where he reached a maximum depth of about $125 \mathrm{~m}$ (data not shown). He did not produce any combined tonal/pulsed signals or whistles for the first 24 dives that exceeded roughly $10 \mathrm{~m}$ following his release, a response more closely resembling the silent reaction observed and discussed by Finley et al. (1990) of narwhals exposed to envi-

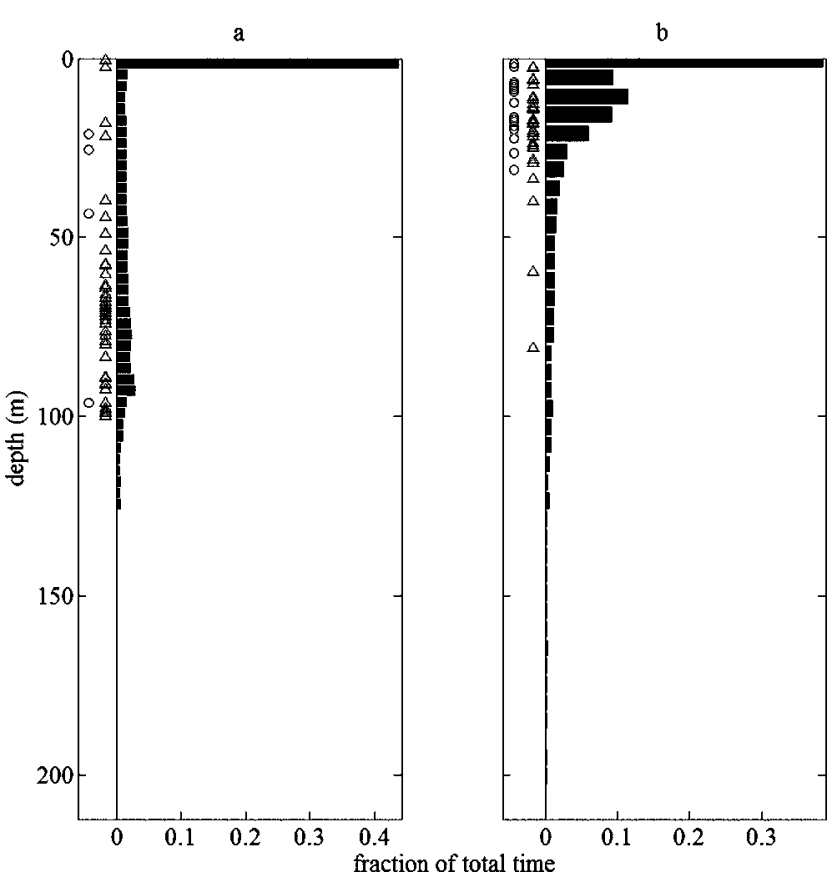

FIG. 7. Approximate depths where combined tonal/pulsed signals (triangles, $\triangle$ ) and whistles (circles, $\bigcirc$ ) were produced adjacent to a frequency histogram of depth bins (bars) for $\mathrm{mm} 224$ (a) and $\mathrm{mm} 226$ (b). The frequency plotted on the abscissa is expressed as a fraction of the total amount of time spent at all depths. The maximum depths achieved for $\mathrm{mm} 224$ and $\mathrm{mm} 226$ during the DTAG deployments were roughly 125 and $210 \mathrm{~m}$, respectively. 

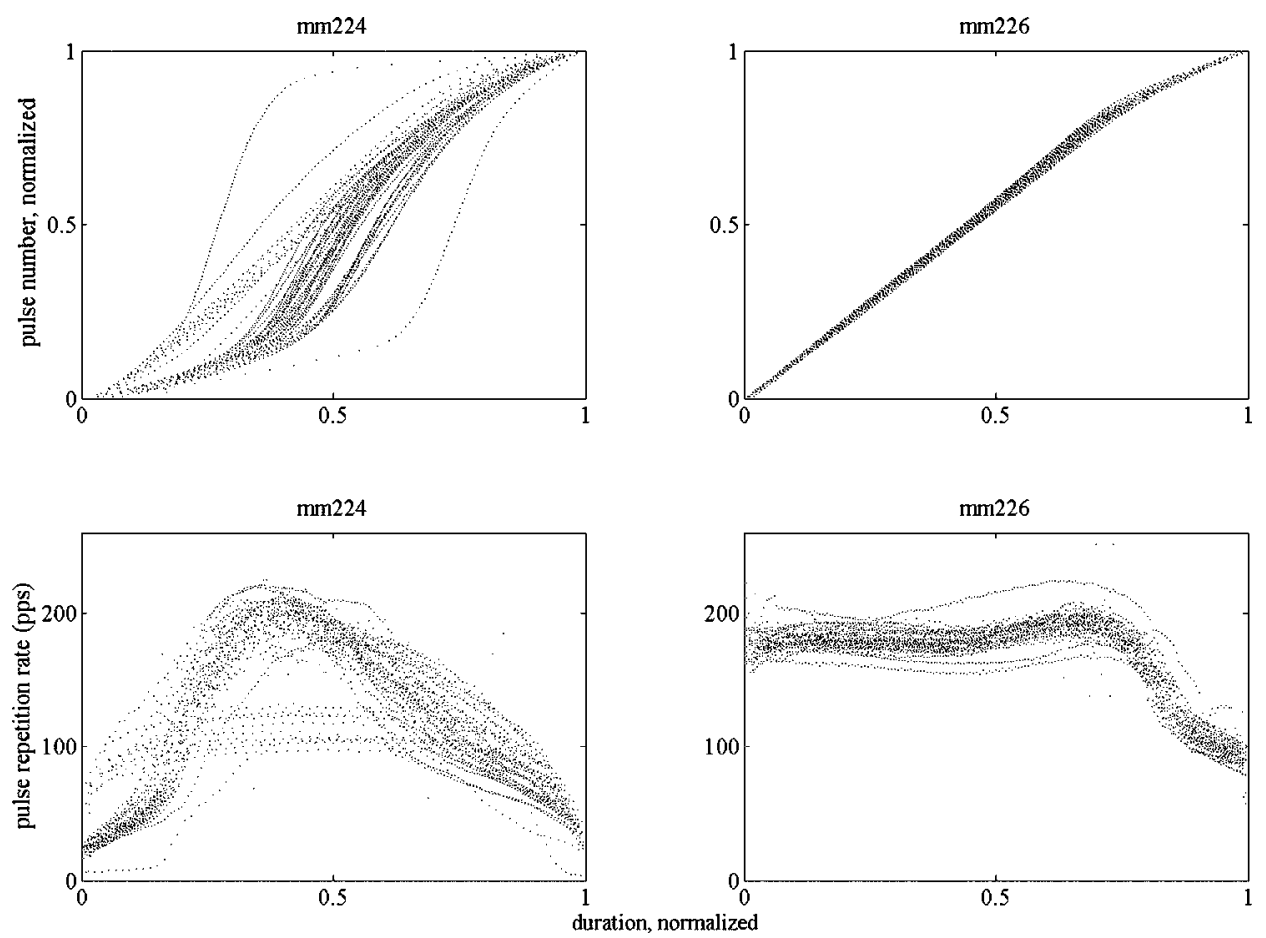

FIG. 8. Visual representations of temporal features of combined tonal/ pulsed signals. Normalized pulse number (top two panels) and pulse repetition rate (pulses per second, bottom two panels) as a function of normalized duration. Note the clear differences in general morphology of these plots between the two individuals. ronmental disturbances. Whistles were less common than combined tonal/pulsed signals, as reported in earlier studies (Ford and Fisher, 1978; Miller et al., 1995).

The combined tonal/pulsed signals lasted between 0.55 and 2.68 s and contained between 49 and 202 pulses. Spectrograms revealed the synchronous production of both pulsatile energy in the form of repeated broadband impulses and a tonal, FM component by the tagged animals (Fig. 2). The FM component was not an analytical artifact of the pulsatile energy (see Watkins, 1967) because the fundamental frequency of the tonal feature was inconsistent with the repetition rate of the pulses. It is likely that at least two sound generating apparatuses are required to produce these combined tonal/pulsed signals to achieve pulsatile and FM energy content simultaneously. Combined tonal/pulsed signals were characterized by pulse rates between 28 and 113 pulses/s for mm224 and between 148 and 180 pulses/s for $\mathrm{mm} 226$. Figure 2 reveals additional low frequency energy associated with each pulse, which is likely the consequence of resonance of the air sacs of the tagged animal and not reverberations from or echoes off of elements along the inlet bottom. Indeed, no echoes consistent with target localization or monitoring position in the water column were detected in the audio record. All whistles were between 0.68 and $1.26 \mathrm{~s}$ with frequencies ranging between 360 and $8844 \mathrm{~Hz}$. Three of the four whistles produced by $\mathrm{mm} 224$ were characterized by a brief $(0.18-0.24 \mathrm{~s})$ upsweep, followed by a pause and longer (0.62-0.66 s) downsweep [Figs. 3(a), 3(b), and 3(d)]. The fourth whistle was continuous but still showed an upsweep preceding the downsweep [Fig. 3(c)]. All seventeen whistles produced by mm226 contained a brief (0.026-0.091 s) broadband segment with energy that peaked between about 500 and $700 \mathrm{~Hz}$ and then decayed steadily until disappearing above $8-10 \mathrm{kHz}$ followed by a flat, constant frequency tone that lasted for the remainder of the whistle [Figs. 3(f)-3(s), 3 whistles are not shown]. The whistles were all of about the same intensity except for a quieter one recorded on the tag attached to mm226 [Fig. 3(j)], which may have been softer, may have been produced by a nonfocal animal located further from the tag, or may have had a transmission path that was partially obscured by the animal or tag components before reaching the hydrophone.

The uniqueness of each of the two sets of combined tonal/pulsed signals and whistles was apparent from simple visual inspection. Among the combined tonal/pulsed signals the patterns of how the relative timing and repetition rate of the pulses varied as a function of normalized duration differed between the two individuals (Fig. 8). All measured features for both the combined tonal/pulsed signals and whistles differed significantly between the two animals (Wilcoxon rank sum test with a Bonferroni correction, $P=0.002$ for combined tonal/pulsed signals duration, $P<0.001$ for remaining combined tonal/pulsed signals measurements, $P$ $=0.011$ for whistle ending frequency and $P=0.008$ for remaining whistle measurements). The whistles produced by mm224 were longer and higher in every measurement compared to those belonging to $\mathrm{mm} 226$, which were shorter and lower. Indeed, the minimum frequency of mm224 was $1549 \pm 201 \mathrm{~Hz}(\bar{x} \pm \mathrm{sd})$ and the maximum frequency of mm226 was $1177 \pm 111 \mathrm{~Hz}(\bar{x} \pm \mathrm{sd})$, values that did not overlap even a single standard deviation away (Table II). The cross-correlation test on the whistles revealed dramatic differences for the interindividual comparisons (between mm224 and mm226) and only slight differences among the intraindividual comparisons (Table III). The interindividual results were more different than the intraindividual results by 1-2 orders of magnitude. 
TABLE III. Cross-correlation comparison of whistles between the same and different individuals. These data were computed in arbitrary units with higher values indicating a greater difference between the contours being compared. The intraindividual comparisons are italicized.

\begin{tabular}{lrr}
\hline \hline & $\mathrm{mm} 226$ & $\mathrm{~mm} 224$ \\
\hline $\mathrm{mm} 224$ & 127250 & 5348 \\
$\mathrm{~mm} 226$ & 716 & \\
\hline \hline
\end{tabular}

\section{DISCUSSION AND CONCLUSIONS}

Two free-ranging narwhals each produced an acoustically distinctive set of combined tonal/pulsed signals and whistles. Visual and aural inspection and nonparametric and cross-correlation analyses all demonstrated striking interindividual differences among these vocalizations and intraindividual temporal and spectral fidelity. These results support the claim of Ford and Fisher (1978) that narwhals produce individually distinctive signature vocalizations. The recordings analyzed here also contained numerous faint combined tonal/pulsed signals and whistles produced by nonfocal animals. These observations are consistent with the conclusion that these vocal categories are regularly produced by freeranging narwhals in this area.

The function of these vocalizations remains uncertain, but they do not appear to facilitate foraging. When feeding, some odontocetes produce a sequence of regularly spaced echolocation clicks that precede a buzz, or a series of clicks characterized by a dramatically elevated repetition rate [e.g., sperm whales (Physeter macrocephalus): Miller et al., 2004, Blainville's beaked whales (Mesoplodon densirostris): Madsen et al., 2005]. It seems unlikely that the narwhal vocalizations quantified here were used for foraging purposes since no echolocation clicks were detected immediately before the combined tonal/pulsed signals or the whistles. Indeed, the kind of clicking behavior characterized by changes in repetition rate and amplitude that is associated with foraging has been recorded from narwhals in previous studies (see Møhl et al., 1990; Miller et al., 1995), but was not observed here.

Combined tonal/pulsed signals and whistles may play a role in social communication based on their stereotypy (Ford and Fisher, 1978) and the signature content shown by the limited dataset presented here. These distinctive vocalizations might serve as contact calls to facilitate reunions of individuals with their group members in a manner similar to that observed in captive and free-ranging bottlenose dolphins (Janik and Slater, 1998; Watwood et al., 2005). Unlike the studies conducted with bottlenose dolphins to identify pair bonds or alliances among males (Connor et al., 1992, 2001; reviewed in Wells, 2003), little work has been completed to describe the social structure and group relationships among individual narwhals. Based on personal observations, the narwhals entering Admiralty Inlet traveled in groups ranging in size from roughly 5 to 30 animals. The group members traveling with the tagged animals vacated the area while their companions were detained on shore. The vocalizations of more distant animals that were recorded in this study were usually faint, suggesting that for the tagged animals, the dives occurring after their capture were likely solitary events. In addition, no other animals appeared in accompanying video footage recorded from a Crittercam (with a visual range extending between 3 and $20 \mathrm{~m}$ depending on the light level) attached to mm226 during the first hour following its release. These observations are consistent with the hypothesis that the combined tonal/pulsed signals and/or whistles were used by these two narwhals as contact calls in an effort to regain contact with their groups. However, actual reunions with other animals were not obvious from the acoustic record.

Because these two animals were likely members of different groups traveling into Admiralty Inlet, an alternative explanation consistent with the results presented here is that these vocalization classes may have been distinctive at the level of the social group (see Terhune et al., 2001; Weiß et al., 2006). Another possibility is that combined tonal/pulsed signals and whistles are actually used as signature vocalizations to cue conspecifics about individual identity. The whistle of mm226 was characterized by nearly constant frequency except for the brief noisy segment at the very beginning. From an information theory perspective, a flat whistle encodes less information compared to a frequency modulated whistle. It is possible, however, that even flat whistles of consistently distinctive durations or pitches could be used to distinguish between individuals. The contours of the whistles of mm226 appeared very similar to the FM component of the combined tonal/pulsed signals of this animal. The whistles produced by $\mathrm{mm} 224$, however, contained the frequency modulation expected of signature vocalizations and did not resemble this animal's combined tonal/pulsed signals FM component.

The combined tonal/pulsed signals described here most closely resembled the longer click series and the pulsed sounds described by Watkins et al. (1971) and Ford and Fisher (1978), respectively. All of these vocalizations were characterized by a combination of pulses and a tonal signal. Watkins et al. (1971) described the repetition rate of their longer click series tending to increase before becoming constant and eventually slowing down, somewhat similar to the trend observed in the combined tonal/pulsed signals described here (Fig. 8). In both the combined tonal/pulsed signals recorded in this study and their equivalents described in Watkins et al. (1971) and Ford and Fisher (1978), the synchronously-produced FM component creating the tonal quality in these sounds was not due exclusively to harmonically-related sidebands of the repetition rate (see Watkins, 1967). Note in Fig. 2 that the pitch of the FM component does not always correspond to the repetition rate of the pulses. For the combined tonal/pulsed signals produced by $\mathrm{mm} 226$, for example, the FM component begins over halfway through the signal without any observable change in repetition rate. Also, the tonal energy persists even as the pulses slow down at the end of the combined tonal/ pulsed signals attributed to individual mm224 [Fig. 2(a)] and continues beyond the conclusion of the pulses in the signal assigned to mm226 [Fig. 2(b)]. The pulsatile component of 
the combined tonal/pulsed signals quantified here was characterized by a higher upper frequency limit (up to $48 \mathrm{kHz}$ ) compared to earlier recordings.

Previous studies (Watkins et al., 1971; Ford and Fisher, 1978) reported observing tonal signals with properties that were both similar to and different from the whistles analyzed here. The frequency ranges overlapped but the whistles that were recorded here had higher harmonics, extending the upper bound of these tonal vocalizations to at least $48 \mathrm{kHz}$. Earlier published tones were either constant in frequency or swept upwards or downwards, again consistent with the whistles presented here (Fig. 3). Both earlier works, however, described whistles as narrow-band signals lacking additional detectable harmonic content. All of the whistles recorded for this study were typified by a fundamental FM component and harmonic energy, a difference which may have resulted from the higher sampling rate of the recording equipment and/or the elevated signal to noise ratio due to the close proximity of the hydrophone to the whale.

The cross-correlation test used in this analysis was modified slightly from those described in other studies of signature vocalizations (e.g., Buck and Tyack, 1993; McCowan, 1995; Janik, 1999; Watwood et al., 2005). In general, similarity between whistle contours can result either coarsely from a general overlap in frequency range and/or more finely from comparable frequency modulation (e.g., loop number, overall shape). The time-invariant crosscorrelation test used here incorporated both of these components into its final measurement. A continuum was possible ranging from dissimilar (minimal frequency overlap and contour resemblance) to very similar (maximal frequency overlap and contour resemblance). Because the first term of the product ranked similarity according to both overlap and contour and the second term according to contour only, intermediate scores of similarity were also possible. This was particularly important since the frequency ranges of the whistles from the two narwhals were mostly nonoverlapping. The possibility of contour shape resemblance was excluded by the unambiguous results of the cross-correlation test (Table III).

These findings suggest possible directions for future work. Tagging and recording the combined tonal/pulsed signals and whistles of multiple narwhals from other groups would provide data that could support or reject the conclusions made here. If these signals do possess signature content, further study could ascertain whether they are distinctive at the individual or group level. Critical to determining the function of these vocalizations will be an assessment of the behavioral context in which these vocalizations are produced and the stability of group composition and size over short and long time scales. If narwhals are capable of differentiating between individuals acoustically, quantifying these aspects of group dynamics would provide starting estimates for the number of animals with which a single individual is interacting and therefore between which it should be able to distinguish. Playback experiments would be useful for identifying the temporal and spectral features of the combined tonal/pulsed signals and whistles that the animals may be using to facilitate differentiation. An understanding of the ontogeny of these sounds to determine if vocal learning plays any role in their acquisition or development requires acoustic data from the same animals and their groups collected longitudinally over many years.

Signature whistles appear to be used by bottlenose dolphins as contact calls in a variety of contexts (Janik and Slater, 1998; Sayigh et al., 1998; Watwood et al., 2005). If narwhals, another gregarious odontocete, similarly use their combined tonal/pulsed signals and whistles as contact calls when separated from conspecifics, the procedure described here affords an opportunity to make recordings in this context while the animals are detained ashore. Under this hypothesis, an involuntary separation of the sort imposed here would cause the animals and/or their group members to vocalize in an effort to regain contact.

Despite the small sample size, the data presented in this manuscript provide supportive evidence for at least two classes of signature vocalizations among free-ranging narwhals at the individual or group level. Future work focused on the ontogeny, function, and acoustic characteristics of the combined tonal/pulsed signals and whistles produced by narwhals is required to develop an improved understanding of the vocal and social behavior of this elusive Arctic animal.

\section{ACKNOWLEDGMENTS}

This manuscript benefited from the insights, thorough revisions, and helpful suggestions provided by Peter Tyack. Special thanks to Mark Johnson, Kira Barton, Alex Bocconcelli, Amanda Hansen, Tom Hurst, and Alex Shorter for DTAG and field support and to Rune Dietz, Jack Orr, Mehdi Bakhtiari, Sila Akikuluk, Sandie Black, Moe Keenainak, Martin Nweeia, Hans-Christian Schmidt, Seemee Tunraq, and Keith Yip for their generous assistance in the field. I am grateful to Amanda Searby and Stephanie Watwood for comments on the contour comparisons and to two anonymous reviewers whose suggestions helped to improve this manuscript. I thank the WHOI Academic Programs office, the National Science Foundation Research Fellowship, and the National Defense Science and Engineering Graduate Fellowship for financial support. This field operation was funded by the Greenland Institute of Natural Resources, the National Environmental Research Institute, Department of Fisheries and Oceans, Nunavut Wildlife Management Board and the Danish Cooperation for the Environment in the Arctic (DANCEA). Additional logistical support was provided by the Polar Continental Shelf Project. This work was conducted under permit No. SLE-04/05-9 issued by the Canadian Department of Fisheries and Oceans and animal use protocol FWI2004-2005-10.

Balcombe, J. P. (1990). "Vocal recognition of pups by mother Mexican free-tailed bats, Tadarida brasiliensis mexicana," Anim. Behav. 39, 960-966.

Bee, M. A., and Gerhardt, H.C. (2002). "Individual voice recognition in a territorial frog (Rana catesbeiana),” Proc. R. Soc. London, Ser. B 269, 1443-1448.

Boughman, J. W. (1997). "Greater spear-nosed bats give group-distinctive calls," Behav. Ecol. Sociobiol. 40, 61-70.

Buck, J. R., and Tyack, P.L. (1993). "A quantitative measure of similarity for Tursiops truncatus signature whistles," J. Acoust. Soc. Am. 94, 2497-2506. 
Caldwell, M. C., and Caldwell, D.K. (1965). "Individualized whistle contours in bottlenose dolphins (Tursiops truncatus)," Nature (London) 207, 434-435.

Charrier, I., Mathevon, N., and Jouventin, P. (2001). "Mother's voice recognition by seal pups," Nature (London) 412, 873.

Charrier, I., Mathevon, N., and Jouventin, P. (2003). "Vocal signature recognition of mothers by fur seal pups," Anim. Behav. 65, 543-550.

Connor, R. C., Heithaus, M. R., and Barre, L. M. (2001). "Complex social structure, alliance stability and mating access in a bottlenose dolphin 'super-alliance'," Proc. R. Soc. London, Ser. B 268, 263-267.

Connor, R. C., Smolker, R. A., and Richards, A. F. (1992). "Two levels of alliance formation among male bottlenose dolphins (Tursiops sp.)," Proc. Natl. Acad. Sci. U.S.A. 89, 987-990.

Deecke, V. B., Ford, J. K. B., and Spong, P. (2000). "Dialect change in resident killer whales: implications for vocal learning and cultural transmission," Anim. Behav. 60, 629-638.

Dietz, R., and Heide-Jørgensen, M. P. (1995). "Movements and swimming speed of narwhals Monodon monoceros, equipped with satellite transmitters in Melville Bay, northwest Greenland," Can. J. Zool. 73, 2106-2119.

Dietz, R., Heide-Jørgensen, M. P., Richard, P. R., and Acquarone, M. (2001). "Summer and fall movements of narwhals (Monodon monoceros) from northeastern Baffin Island towards northern Davis Strait," Arctic 54, 244-261.

Egnor, S. E. R., andHauser, M. D. (2004). "A paradox in the evolution of primate vocal learning," Trends Neurosci. 27, 649-654.

Elowson, A. M., andSnowdon, C. T. (1994). "Pygmy marmosets, Cebuella pygmaea, modify vocal structure in response to changed social environment," Anim. Behav. 47, 1267-1277.

Farabaugh, S. M., Linzenbold, A., and Dooling, R. J. (1994). "Vocal plasticity in budgerigars (Melopsittacus undulatus) -Evidence for social factors in the learning of contact calls," J. Comput. Phys. 108, 81-92.

Finley, K. J., Miller, G. W., Davis, R. A., and Greene, C. R. (1990). "Reactions of belugas, Delphinapterus leucas, and narwhals, Monodon monoceros, to ice-breaking ships in the Canadian high Arctic," Can. Bull. Fish. Aquat. Sci. 224, 97-117

Ford, J. K. B., and Fisher, H. D. (1978). "Underwater acoustic signals of the narwhal (Monodon monoceros)," Can. J. Zool. 56, 552-560.

Fripp, D., Owen, C., Quintana-Rizzo, E., Shapiro, A., Buckstaff, K., Jankowski, K., Wells, R., and Tyack, P. (2005). "Bottlenose dolphin (Tursiops truncatus) calves appear to model their signature whistles on the signature whistles of community members," Anim. Cogn. 8, 17-26.

Frommolt, K. H., Goltsman, M. E., and MacDonald, D. W. (2003). "Barking foxes, Alopex lagopus: Field experiments in individual recognition in a territorial mammal," Anim. Behav. 65, 509-518.

Goldman, J. A., Phillips, D. P., and Fentress, J. C. (1995). "An acoustic basis for maternal recognition in timber wolves (Canis lupus)," J. Acoust. Soc. Am. 97, 1970-1973.

Hay, K. A., and Mansfield, A. W. (1989). "Narwhal: Monodon monoceros Linnaeus, 1758," in Handbook of Marine Mammals: Volume 4, River Dolphins and the Larger Toothed Whales, edited by S. H. Ridgway and R. Harrison (Academic, London), pp. 145-176.

Janik, V. M. (1999). "Pitfalls in the categorization of behaviour: a comparison of dolphin whistle classification methods," Anim. Behav. 57, 133143.

Janik, V. M. (2000). "Whistle matching in wild bottlenose dolphins (Tursiops truncatus)," Science 289, 1355-1357.

Janik, V. M., Sayigh, L. S., and Wells, R. S., (2006). "Signature whistle shape conveys identity information to bottlenose dolphins," Proc. Natl. Acad. Sci. U.S.A. 103, 8293-8297.

Janik, V. M., and Slater, P. J. B. (1997). "Vocal learning in mammals," Adv Study Behav. 26, 59-99.

Janik, V. M., and Slater, P. J. B. (1998). "Context-specific use suggests that bottlenose dolphin signature whistles are cohesion calls," Anim. Behav. 56, 829-838.

Janik, V. M., and Slater, P. J. B. (2000). "The different roles of social learning in vocal communication," Anim. Behav. 60, 1-11.

Johnson, M. P. and Tyack, P. L. (2003). "A digital acoustic recording tag for measuring the response of wild marine mammals to sound," IEEE J. Ocean. Eng. 28, 3-12.

Jones, B. S., Harris, D. H. R., and Catchpole, C. K. (1993). "The stability of the vocal signature in phee calls of the common marmoset, Callithrix jacchus," Am. J. Primatol 31, 67-75.

Laidre, K. L., Heide-Jørgensen, M. P., Logdson, M. L., Hobbs, R. C., Heagerty, P., Dietz, R., Jørgensen, O. A., and Treble, M. A. (2004). "Seasonal narwhal habitat associations in the high Arctic," Mar. Biol. (Berlin) 145, 821-831.

Lengagne, T., Aubin, T., Jouventin, P., and Lauga, J. (2000). "Perceptual salience of individually distinctive features in the calls of adult king penguins," J. Acoust. Soc. Am. 107, 508-516.

Leonard, M. L., Horn, A. G., Brown, C. R., and Fernandez, N. J. (1997). "Parent-offspring recognition in tree swallows, Tachycineta bicolor," Anim. Behav. 54, 1107-1116.

Madsen, P. T. (2005). "Marine mammals and noise: Problems with root mean square sound pressure levels for transients," J. Acoust. Soc. Am. 117, 3952-3957.

Madsen, P. T., Johnson, M., de Soto, N. A., Zimmer, W. M. X., and Tyack, P. (2005). "Biosonar performance of foraging beaked whales (Mesoplodon densirostris)," J. Evol. Biol. 208, 181-194.

Marshall, A. J., Wrangham, R. W., and Arcadi, A. C. (1999). "Does learning affect the structure of vocalizations in chimpanzees?," Anim. Behav. 58, $825-830$.

Marshall, G. J. (1998). "Crittercam: An animal-borne imaging and data logging system," Mar. Technol. Soc. J. 32, 11-17.

McCowan, B. (1995). "A new quantitative technique for categorizing whistles using simulated signals and whistles from captive bottlenose dolphins (Delphinidae, Tursiops truncatus)," Ethology 100, 177-193.

McCowan, B. and Reiss, D. (1995). "Quantitative comparison of whistle repertoires from captive adult bottlenose dolphins (Delphinidae, Tursiops truncatus)-A re-evaluation of the signature whistle hypothesis," Ethology 100, 194-209.

McCowan, B., and Reiss, D. (2001). "The fallacy of 'signature whistles' in bottlenose dolphins: A comparative perspective of 'signature information' in animal vocalizations," Anim. Behav. 62, 1151-1162.

Miksis, J. L., Tyack, P. L., and Buck, J. R. (2002). "Captive dolphins, Tursiops truncatus, develop signature whistles that match acoustic features of human-made model sounds," J. Acoust. Soc. Am. 112, 728-739.

Miller, L. A., Pristed, J., Møhl, B., and Surlykke, A. (1995). "The clicksounds of narwhals (Monodon monoceros) in Inglefield Bay, Northwest Greenland," Marine Mammal Sci. 11, 491-502.

Miller, P. J. O., and Bain, D. E. (2000). "Within-pod variation in the sound production of a pod of killer whales, Orcinus orca," Anim. Behav. 60, 617-628.

Miller, P. J. O., Johnson, M. P., and Tyack, P. L. (2004). "Sperm whale behaviour indicates the use of echolocation click buzzes 'creaks' in prey capture," Proc. R. Soc. London, Ser. B 271, 2239-2247.

Mitani, J. C., and Gros-Louis, J. (1998). "Chorusing and call convergence in chimpanzees: Tests of three hypotheses," Behaviour 135, 1041-1064.

Mitani, J. C. and Nishida, T. (1993). "Contexts and social correlates of long distance calling by male chimpanzees," Anim. Behav. 45, 735-746.

Møhl, B., Surlykke, A., and Miller, L. A. (1990). "High intensity narwhal clicks," in Sensory Abilities of Cetaceans, edited by J. Thomas and R. Kastelein (Plenum, New York), pp. 295-303.

Nousek, A. E., Slater, P. J. B., and Miller, P. J. O. (in press). "The influence of social affiliation on individual vocal signatures of northern resident killer whales (Orcinus orca)," Proc. R. Soc. London, Ser B.

Sayigh, L. S., Tyack, P. L., Wells, R. S., and Scott, M. D. (1990). "Signature whistles of free-ranging bottenose dolphins, Tursiops truncatus: Motheroffspring comparisons," Behav. Ecol. Sociobiol. 26, 247-260.

Sayigh, L. S., Tyack, P. L., Wells, R. S., Solow, A. R., Scott, M. D., and Irvine, A. B. (1998). "Individual recognition in wild bottlenose dolphins: a field test using playback experiments," Anim. Behav. 57, 41-50.

Scherrer, J. A., and Wilkinson, G. S. (1993). "Evening bat isolation calls provide evidence for heritable signatures," Anim. Behav. 46, 847-860.

Searby, A., Jouventin, P., and Aubin, T. (2004). "Acoustic recognition in macaroni penguins: An original signature system," Anim. Behav. 67, $615-625$.

Smolker, R., and Pepper, J. W. (1999). "Whistle convergence among allied male bottlenose dolphins (Delphinidae, Tursiops sp.)," Ethology 105, 595-617.

Snowdon, C. T., and Elowson, A. M. (1999). "Pygmy marmosets modify call structure when paired," Ethology 105, 893-908.

Snowdon, C. T., Elowson, A. M., and Roush, R. S. (1997). "Social influences on vocal development in New World primates," in Social Influences on Vocal Development, edited by C. T. Snowdon and M. Hausberger (Cambridge University Press, Cambridge), pp. 234-248.

Stoddard, P. K., and Beecher, M. D. (1983). "Parental recognition of offspring in the cliff swallow," Auk 100, 795-799.

Terhune, J. M., Healey, S. R., and Burton, H. R. (2001). "Easily measured 
call attributes can detect vocal differences between Weddell seals from two areas," Bioacoustics 11, 211-222.

Tyack, P. (1986). "Whistle repertoires of two bottlenosed dolphins, Tursiops truncatus: Mimicry of signature whistles?" Behav. Ecol. Sociobiol. 18, 251-257.

Watkins, W. A. (1967). "The harmonic interval: fact or artifact in spectral analysis of pulse trains," in Marine Bioacoustics, edited by W. N. Tavolga (Pergamon, New York), pp. 15-43.

Watkins, W. A., Schevill, W. E., and Ray, C. (1971). "Underwater sounds of Monodon (Narwhal)," J. Acoust. Soc. Am. 49, 595-599.

Watwood, S. L., Owen, E. C. G., Tyack, P. L., and Wells, R. S. (2005).

"Signature whistle use by temporarily restrained and free-swimming bottlenose dolphins, Tursiops truncatus," Anim. Behav. 69, 1373-1386. Watwood, S. L., Tyack, P. L., and Wells, R. S. (2004). "Whistle sharing in paired male bottlenose dolphins, Tursiops truncatus," Behav. Ecol. Sociobiol. 55, 531-543.

Weiß, B. M., Ladich, F., Spong, P., and Symonds, H. (2006). "Vocal behavior of resident killer whale matrilines with newborn calves: The role of family signatures,” J. Acoust. Soc. Am. 119, 627-635.

Wells, R. S. (2003). "Dolphin Social Complexity: Lessons from Long-Term Study and Life History," in Animal Social Complexity: Intelligence, Culture, and Individualized Societies, edited by F. B. M. de Waal and P. L. Tyack (Harvard University Press, Cambridge, Massachusetts), pp. 32-56. 